This is the accepted version of the following article:

Booker, Liesel and Mullan, Barbara. 2013. Using the temporal self-regulation theory to examine the influence of environmental cues on maintaining a healthy lifestyle. British Journal of Health Psychology. 18 (6): pp. 745-762., which has been published in final form at http://doi.org/10.1111/bjhp.12015 
Running head: Self-regulation, environmental cues, and healthy lifestyle

\section{Using the temporal self-regulation theory to examine the influence of environmental cues on maintaining a healthy lifestyle}

Liesel Booker ${ }^{1}$ and Barbara Mullan ${ }^{1 *}$

${ }^{1}$ School of Psychology, University of Sydney

Word count (exc. figures/tables): 4925

${ }^{*}$ Requests for reprints should be addressed to Barbara Mullan, School of Psychology, University of Sydney, NSW 2006, Australia (e-mail: barbara.mullan@sydney.edu.au). 


\section{Statement of contribution}

\section{What is already known on this subject?}

- Young adults fail to adhere to behaviours indicative of healthy lifestyle.

- Self-regulation and behavioural prepotency add unique variance to the prediction of health behaviour. The influence of these factors is thought to vary according to environmental context.

\section{What does this study add?}

- Individuals who feel supported by the environment are more likely to maintain a healthy lifestyle than those who feel distracted by the environment.

- Behavioural prepotency is predictive of healthy lifestyle for individuals who feel 'supported' by the environment.

- Behavioural prepotency, planning and response inhibition are predictive of healthy lifestyle for individuals who feel 'unsupported' by the environment. 
Objectives: The aim of the current study is to explore the predictive utility of the Temporal Self-Regulation Theory (TST) for maintaining a healthy lifestyle (Hall \& Fong, 2007). According to TST, the influence of intention, self-regulation and behavioural prepotency differs depending on the environmental context in which the behaviour is performed. This study examined the influence of perceptions about the supportiveness of the environmental context on TST related factors.

Design: TST was tested using a prospective design with one week follow-up.

Methods: One hundred and fifty-two undergraduates were administered three executive functioning tasks and an online questionnaire regarding their intentions to maintain a healthy lifestyle, environmental responsiveness and previous behaviour. One week later they completed a follow-up questionnaire.

Results: Participants who were supported by the environment were significantly more likely to maintain a healthy lifestyle than those distracted by the environment. Behavioural prepotency was significantly predictive of behaviour performance for 'supported' participants. Behavioural prepotency, planning and response inhibition were significantly predictive of 'unsupported' participants' behaviour.

Conclusions: These findings provided preliminary support for the use of TST for the prediction of healthy lifestyle behaviour. Importantly, this study provided support for the contention that the influence of TST related factors would vary according to the perceived supportiveness of the environment. These findings suggest that environmental responsiveness may be an important determinant to close the intentionbehaviour gap for maintaining a healthy lifestyle. 


\section{Introduction}

The link between health behaviours, lifestyle and outcomes, such as quality of life and mortality, has been widely publicised and replicated (Berkman \& Syme, 1979; Brock, Haefner, \& Noble, 1988; Kaplan, Baltrus, \& Raghunathan, 2007). The Alameda study established that better health outcomes were associated with common health behaviours, often referred to as the 'Alameda 7,' including never smoking, drinking in moderation, sleeping between 7 and 8 hours per night, exercising, maintaining a healthy weight, avoiding snacking and regularly consuming breakfast (Belloc \& Breslow, 1972). Further evidence for the link between lifestyle and health comes from a World Health Organisation report (WHO, 2011) which suggests that non-communicable diseases (NCDS) including cardiovascular disease, cancers, diabetes and chronic respiratory diseases are responsible for $63 \%$ of global deaths. The report also stated that these deaths could be largely prevented through the reduction of four health-risk behaviours: tobacco use, alcohol abuse, physical inactivity and unhealthy diet.

Maintaining a healthy lifestyle is likely to be affected by intrinsic influences such as personal and motivational factors as well as extrinsic factors including the context and availability of behavioural/lifestyle choices. Understanding the behavioural and psychosocial factors influencing lifestyle choices as well as of the barriers that prevent people from adopting or maintaining a healthy lifestyle (Milligan et al., 1998) is critical for developing health promotion interventions. Theoretical models provide a framework for developing an understanding of such factors (Lippke \& Ziegelmann, 2008; Michie, Rothman, \& Sheeran, 2007), and traditional social-cognitive models such the Theory of Planned Behaviour, (Ajzen, 1991) have been widely utilised to explore predictive factors for health behaviours (Hall \& Fong, 2007). Such theories assume behaviour to be largely rational and driven by an individual's intentions (Webb \& Sheeran, 2006). Intentions reflect motivation and are 
indicative of the effort an individual will apply to performing the behaviour (Ajzen, 1991; Webb \& Sheeran, 2006). However, while intentions have been demonstrated as important predictors of health behaviour (e.g. Armitage \& Conner, 2001), the intention-behaviour relationship is imperfect. Thus, identifying factors to account for this disparity is important as people frequently fail to act upon their intentions to perform health behaviours (Abraham et al., 1999).

One recent theory that attempts to address this is Hall and Fong's (2007) Temporal Self-Regulation Theory (TST), which recognises that the temporal dispersion of costs and valences for health behaviours affects the individual's motivational level and may therefore explain the inconsistency in the intention-behaviour relationship. Most health-protective behaviours such as exercise and healthy eating are typified by immediate negative contingencies such as effort or discomfort but are beneficial in the long-term if performed consistently. Health-risk behaviours such as smoking are often immediately satisfying or pleasurable but their impact on long-term health is costly. As decision-making is commonly influenced by immediate contingencies rather than long-term outcomes, an individual's motivation to engage in or avoid particular behaviours is arguably moderated by these factors. TST purports that as the temporal disparity between costs and valence increases for health behaviours, so too does the influence of post-intentional constructs such as behavioural prepotency and self-regulation on behavioural performance, as intention may not be sufficient for behavioural performance (see Figure 1).

Insert Figure 1 near here

Behavioural prepotency represents the behavioural response that takes precedence over all other responses (Hall \& Fong, 2007). Prepotent responses are pervasive in everyday life and dictated by internal or environmental cues, biological disposition and past behaviour (Sallis, 2010). By their nature, prepotent responses are triggered without conscious 
awareness. Behavioural prepotency is thought to influence future behaviour both directly and indirectly through moderating the intention-behaviour relationship (Ouellette \& Wood, 1998; Sheeran \& Abraham, 2003).

It has been suggested that in the TST the frequency of past behaviour can be used as a proxy for behavioural prepotency (Hall \& Fong, 2007). Past behaviour consistently appears to be the best single predictor of future behaviour (Ouellette \& Wood, 1998), as shown in studies into breakfast consumption (Wong \& Mullan, 2009), physical activity (Kwan, Bray, \& Ginis, 2009) and sleep hygiene (Kor \& Mullan, 2011). Previous health behaviour research found that while intention had been a significant predictor of behaviour initially, intention lost its predictive utility when the model included past behaviour (Collins \& Mullan, 2011; Hall \& Fong, 2007).

Evidence increasingly suggests that self-regulation, defined as the capacity to manage and control cognitive, behavioural and emotional responses to internal or environmental cues (Baumeister, Schmeichel, \& Vohs, 2007), is also an important component of health behaviour theory (Hall \& Fong, 2007). Adherence to healthy lifestyle behaviours requires planning, the ability to adapt to changes in the environment, and the ability to inhibit responses to unhealthy or unsupportive cues in the environment such as the easy availability of unhealthy food - these are all contained with the concept of self-regulation (Suchy, 2009). Cognitive tests of executive functioning are considered to be appropriate measures of self-regulation (Hall \& Fong, 2010). Aspects of neurocognitive functioning have accounted for additional variance in the intention-behaviour relationship (Hall, Elias, \& Crossley, 2006). Specifically, executive function tests such as the Go/ No-Go, Tower of London and Iowa Gambling Task have been shown to predict unique variance in a variety of health behaviours including dietary and exercise behaviours (Hall, et al., 2006), sleep (Kor \& Mullan, 2011), breakfast (Wong \& Mullan, 2009) and alcohol consumption (Mullan, Wong, Allom, \& Pack, 2011). 
In addition, the TST purports that the influences on the intention-behaviour relationship differ depending on the environmental context present at the time of performance (Hall \& Fong, 2007). If the environment were perceived as being supportive of behavioural performance, then behavioural performance would be less reliant upon intentions and selfregulation than if the same behaviour were performed in an environment that was highly distracting and unsupportive. However, for individuals who experience the immediate environment as unsupportive for behavioural performance, their behaviour is determined by intentions, behavioural prepotency and self-regulation. For example, compared to an individual living independently, one residing with their family may find it easier to eat healthily as residing with parents may increase the availability of nutritious meals and the practice of eating meals at home, reducing the temptation to purchase take-away dinners. For the individual living with their family, eating well would be supported by their environment and therefore would require less motivation and less self-regulation in order to enact their intentions. However, an individual living independently may intend to eat healthily but find this difficult due to the time, effort and cost involved in preparing meals themselves. In order to act upon their intentions to eat well, the individual would need self-regulation to plan and budget for meals and resist the temptation of purchasing take-away despite it being highly accessible and palatable. If there had been previous occasions when the individual made healthy food choices, this may further support their translation of intentions into healthy behavioural choices, as he or she would know they had successfully done this previously.

While the TST highlights the salience of environmental cues to behavioural performance, this remains to be empirically measured (Hall \& Fong, 2010). Individual differences in responsiveness to environmental triggers will arguably determine which variables - self-regulation or behavioural prepotency - are more likely to predict an individual's ability to behave in a manner that is consistent with his or her intentions to 
maintain a healthy lifestyle. Based on the Alameda 7 and WHO (2011) research, this study focuses on six common health behaviours: physical activity, fruit and vegetable $(\mathrm{F} \& \mathrm{~V})$ consumption, breakfast consumption, sleep, alcohol consumption and smoking. Adherence to the national guidelines for these behaviours is arguably reflective of maintaining a 'healthy lifestyle'. Whilst not an exhaustive list of lifestyle factors, the health behaviours chosen have previously been studied in isolation and are considered to be relevant to the developmental stage of undergraduates. For example, in recent research into health behaviours of Australian undergraduates, $88 \%$ of participants failed to consume sufficient F\&V (Allom \& Mullan, 2011), 53\% did not consume breakfast regularly (Wong \& Mullan, 2009), more than a quarter reported binge-drinking (Mullan, et al., 2011; Todd \& Mullan, 2011) and sleep quantity and quality were poor (Kor \& Mullan, 2011). Further, the behaviours included in this study are all repetitive in nature; either they must be repeatedly performed, or alternatively avoided, in order to be beneficial to the individual's health.

Arising from this literature the aims of the current study are to:

1) investigate whether there is an identifiable pattern to the health behaviours of undergraduates;

2) examine the predictive value of the TST for healthy lifestyle performance; and

3) examine if environmental responsiveness differentially determine the individual variables predictive of a healthy lifestyle.

Based on the TST and previous research into the individual health behaviours, it is hypothesised that the predictive utility of TST variables will be differentially reflective of the environmental context. For those who experience the environmental context as supportive of maintaining a healthy lifestyle, self-regulation and behavioural prepotency will be significant predictors of behaviour. In comparison, for those who experience the environmental context 
as unsupportive of maintaining a healthy lifestyle, intentions, self-regulation and behavioural prepotency will be significant predictors of behaviour.

\section{Methods}

\section{Participants}

Two hundred and twenty one undergraduate students were recruited to the study and received course credit for participation. Of these 69 were excluded from the final sample; 27 withdrew before completing the study and 35 experienced technical difficulties. A further 7 were excluded due to exceeding age eligibility criteria. Institution Human Research Ethics approval was obtained.

\section{Measures}

\section{TST variables}

Intention was calculated as the mean of three items (e.g. "I will try to maintain a 'healthy lifestyle' over the next 7 days"), each measured on a 5-point likert scale (1=not at all true of me, $5=$ extremely true of me). Higher intention scores indicate stronger behavioural intention. The scale had a Cronbach's alpha of .91.

Behavioural Prepotency was operationalised as the frequency of past behaviour (Hall \& Fong, 2007). Participants were provided with national guidelines (see Table 1) and asked to indicate on which days during the past week they met these guidelines. Participants were categorised as meeting a guideline if they reported performing the behaviour on 5 or more days within the 7-day period. Participants' behaviour scores were calculated according to the number of behavioural guidelines met (Pearson, Atkin, Biddle, Gorely, \& Edwardson, 2009), culminating in a Healthy Lifestyle Score (HLS range= 0 to 6), with higher scores indicating greater consistency in performing healthy behaviours.

Insert Table 1 near here 
Self-Regulation was measured using three computer-based executive functioning tasks. These were the Tower of London (TOL) (Shallice, 1982), the Go/No-Go (GNG) (Simmonds, Pekar, \& Mostofsky, 2008) and the Iowa Gambling Task (IGT) (Bechara et al. 1994), respectively. The TOL task required that an initial start configuration be transformed into a specific goal state in a minimum of moves. The measure of interest was the participant's mean problem-solving time, with longer times being indicative of better planning (Allan, Johnston, \& Campbell, 2011). The GNG task presented participants with a rectangle which would change colour. Participants were instructed to press the spacebar as quickly as possible when a green rectangle was displayed but to refrain from responding when a blue rectangle was displayed. The measure of interest was the Performance Index $=$ No-Go Accuracy/RTx100, which indicates both performance accuracy and reaction time (Schweiger, Abramovitch, Doniger, \& Simon, 2007). The IGT requires participants to place bets on one of four card decks of differing profitability. Participants were presented with $\$ 2,000$ and informed that their goal was to maximise their winnings. The measure of interest was the final amount of money the participants had remaining after completing the task, with higher earnings indicative of better decision-making (Allom \& Mullan, 2011).

Environmental triggers were calculated as a composite score of five triggers, including physical, sensory, social, internal drives and emotional drives (Todd, Brogan, Mullan, \& Carroll, under review). For example, participants were asked, "Are there any physical triggers in the environment which (positively or negatively) influence you maintaining a 'healthy lifestyle'?" For the triggers the participant reported as being influential, two further questions were asked to determine the environmental trigger's directional effect $(-2=$ distract,$+2=$ promote $)$ and frequency $(1=$ less than weekly, $5=$ several times per day). For each trigger, the trigger score was multiplied by both the directional and frequency scores, to create an environmental responsiveness score. The mean of the five 
environmental responsiveness scores was used as an overall score, indicative of the participant experiencing the environment to be unsupportive or supportive of the maintenance of a healthy lifestyle $(-10$ to +10$)$. A score of 0 was indicative of the participant not noticing an impact of environmental triggers.

Under the TST, Hall and Fong (Hall \& Fong, 2007) described environmental context as being either relatively supportive or relatively unsupportive. Indeed, Hall and Fong show the distinct contribution of different components of the TST, depending on whether or not the environment is supportive. Whilst it is conceivable that environmental context operates on a continuum, the current study focussed on testing the assumptions put forth by Hall and Fong and therefore environmental responsiveness was treated as a dichotomous variable. To create this dichotomy, those scoring $\geq 0$, indicating that the environment has either a neutral or supportive impact on maintaining a healthy lifestyle were categorised as 'supported'; whilst those scoring $<0$, indicating the environment distracted them from maintaining a healthy lifestyle were categorised as 'unsupported'. As a result, 63.8\% $(\mathrm{n}=97)$ of participants reported experiencing the environment to be supportive of maintaining a healthy lifestyle whereas $36.2 \%(\mathrm{n}=55)$ participants reporting it as being unsupportive.

\section{Behaviour}

A replication of the previous behavioural prepotency measure was used to assess adherence to a healthy lifestyle over the past 7 days.

\section{Procedure}

All tasks were computer based and completed online at the participant's choice of time and location.

Part 1 of the study consisted of the executive functioning tasks followed by a questionnaire containing the TST measures and demographic questions. Participants were 
provided with an explanation of a 'healthy lifestyle,' current guidelines for each of the named behaviours and definitions of the pertinent terms.

Part 2 (the behaviour measure) was sent to participants one week after completion of Part 1.

Analysis

Data was analysed using PASW (SPSS) 18.0 for Mac software package. The research hypotheses were tested using correlations, independent samples t-tests and multiple regression analyses.

To examine the predictive value of TST, the TST variables of intention, environmental responsiveness, self-regulation and behavioural prepotency towards a healthy lifestyle were entered in a multiple regression analysis with behaviour as the dependent variable.

Independent samples t-tests were performed to compare the 'supported' and 'unsupported' groups. Further, within both groups, the predictive utility of the TST framework was examined using hierarchical regression analyses.

\section{Results}

\section{Demographic information}

The sample consisted of 152 undergraduate students of which the majority were female $(n=114,75 \%)$. Most participants identified as Asian/Asian-Australian $(n=71,46.7 \%)$ and lived at home with parents $(\mathrm{n}=117,77 \%)$.

\section{Performance of Health Behaviours}

Only $2 \%$ of participants had a HLS of $0,25 \%$ had a score of $1-2,18 \%$ had a score of 3 and the majority $(55 \%)$ received a score of 4 or higher $(\mathrm{M}=3.43, \mathrm{SD}=1.43)$.

The Pearson's correlation matrix in Table 2 shows correlations between the individual behaviours included in the HLS. Physical activity had a small, significant, positive 
correlation with $\mathrm{F} \& \mathrm{~V}$ consumption $(r=.227)$ as well as with breakfast consumption $(r=.278)$. F\&V consumption had a small, significant, positive correlation with breakfast consumption $(r=.281)$. Alcohol had a strong, significant, positive correlation with smoking $(r=.623)$. Insert Table 2 near here

Prediction of behaviour by intention, self-regulation, environmental responsiveness and

\section{behavioural prepotency}

A correlation analysis was first conducted to investigate the association between intention, self-regulation, behavioural prepotency and HLS separately for individuals who found the environment supportive, and those who found the environment unsupportive. In both environments behavioural prepotency was correlated with intention, and behaviour was correlated with both intention and past behaviour (see Table 3). No other correlations were significant.

Insert Table 3 near here.

The predictive utility of intention, environmental responsiveness, self-regulation and behavioural prepotency for HLS was then tested using hierarchical regression analyses. The overall model was statistically significant, accounting for $47.8 \%$ of variance in HLS $\left(R^{2}=\right.$ $\left..478, F_{1,139}=19.54, p<.001\right)$. When holding all other variables constant, behavioural prepotency and environmental responsiveness were both significant predictors of HLS, with scores on behavioural prepotency accounting for an additional $31.2 \%$ of variance in HLS $\left(\Delta R^{2}=.312, p<.001\right)$. Intention, self-regulation and response inhibition were not significant predictors (see Table 3). The executive function tasks were not significantly correlated with one another.

Insert Table 4 near here 


\section{Environmental triggers as determinants of predictive variables}

Independent samples t-tests revealed no significant differences between 'supported' and 'unsupported' participants on TST variables ( $p>.05)$, indicating that they did not significantly differ in ways other than in their responsiveness to environmental triggers.

Three separate hierarchical regression analyses for both 'supported' and 'unsupported' participants were conducted to examine potential differences in the prediction of HLS by the TST variables. In each analysis, intention was entered into the first block, an individual self-regulation measure into the second block and behavioural prepotency into the third block.

\section{Predicting HLS by TST for 'supported' participants}

For all three regression analyses for 'supported' participants, intention was a significant predictor of HLS when entered first into the regression and after the addition of the executive function task (see Table 4). While no executive function task accounted for a significant increase in variance of HSL in either analysis, behavioural prepotency accounted for a significant increase over and above intention and the executive function task, and rendered intention a non-significant predictor of HLS (see Table 4). The three hierarchical regression analyses demonstrate that although intention is a significant predictor of HLS after controlling for self-regulation, only behavioural prepotency was a significant predictor of HLS for 'supported' participants when intention, self-regulation and behavioural prepotency were assessed simultaneously.

Insert Table 5 near here

\section{Predicting HLS by TST for 'unsupported' participants}

Intention was a significant predictor of HLS for all three regression analyses for 'unsupported' participants when entered first into the regression and after the addition of the executive function tasks (see Table 5). Controlling for intention, planning accounted for a 
significant $7.4 \%$ addition of variance in $\operatorname{HLS}\left(R^{2}=.074, F_{1,52}=4.82, p=.032\right)$. Response inhibition and decision making did not account for a significant increase in HLS when controlling for intention ( $p>.05)$. Controlling for intention and planning, behavioural prepotency accounted for a significant $21 \%$ increase of variance in HLS $\left(R^{2}=.21, F_{1,51}=18.11\right.$, $p<.001$; see Table 5). Similarly, response inhibition accounted for a significant addition of $22.5 \%$ of variance in HLS when controlling for intention and response inhibition. Further, controlling for intention and behavioural prepotency, response inhibition was a significant predictor of HLS $(\beta=.277, \mathrm{t}=2.44, \mathrm{p}=.018)$.

Insert Table 6 near here

The results indicate that when intention, self-regulation and behavioural prepotency were assessed simultaneously for the prediction of HLS for 'unsupported' participants, participants with better planning, better response inhibition or more frequent previous adherence to behavioural guidelines were more likely to score a higher HLS whereas better decision making ability does not appear to influence HLS scores.

\section{Discussion}

The aims of this study were to investigate whether the pattern of young adults' behaviours were indicative of maintaining a healthy lifestyle, to examine the predictive value of the TST for healthy lifestyle performance and to examine if environmental responsiveness differentially determined the individual variables predictive of a healthy lifestyle. Three models corresponding to the research aims were developed and tested, revealing that health behaviours do appear to cluster, TST significantly predicted healthy lifestyle maintenance and environmental triggers were important determinants of the maintenance of a healthy lifestyle.

More than half of all of participants reported adherence to at least four behavioural guidelines. Results varied, with very few participants meeting all guidelines and more than 
one quarter adhering to two or less. Although not directly comparable to previous studies due to methodological differences, these results indicate that many participants displayed a similar pattern of health behaviour as found in recent Australian and overseas research (AIFW, 2011; Dodd, Al-Nakeeb, Nevill, \& Forshaw, 2010; Quintiliani, Allen, Marino, KellyWeeder, \& Li, 2010). As expected, physical activity, F\&V consumption and breakfast consumption were all significantly positively correlated (Adams \& Colner, 2008; WHO, 2003). In contrast to overseas research (Keller, Maddock, Hannover, Thyrian, \& Basler, 2008), moderate alcohol consumption was strongly positively correlated with not smoking in the current sample, most likely representative of the current low smoking rates generally in Australia (ABS, 2006).

Consistent with the hypothesis that TST would predict healthy lifestyle performance, this study demonstrated that the model did significantly predict behaviour, accounting for almost half of the variance in behaviour. While account for about $10 \%$ less variance in behaviour than Hall \& Fong's (2007) original finding, these results are consistent with the pattern of results observed in that study. These results are particularly important as the measure encapsulated six separate health behaviours. The inclusion of the post-intentional variables, behavioural prepotency, environmental responsiveness and self-regulation predicted an additional $35 \%$ of variance in healthy lifestyle, with behavioural prepotency and environmental responsiveness each significantly predicting adherence to a healthy lifestyle. Participants who experienced the environment as supportive of a healthy lifestyle and those who had previously maintained a healthy lifestyle had greater success in translating their intentions into behaviour.

Behavioural prepotency alone accounted for $31 \%$ of variance in healthy lifestyle performance. Consistent with other research, behavioural prepotency rendered intention to be a non-significant predictor of behaviour (Collins \& Mullan, 2011), implying that healthy 
lifestyle performance is not entirely under volitional control (Sutton, 1998). This finding supports Hall and Fong's (2007) hypothesis that intention may be of less importance for repetitive behaviour. As the frequency of behavioural prepotency increases, thereby leading to execution of the behaviour without conscious deliberation, the strength of the intentionbehaviour association decreases (Norman \& Conner, 2006).

The influence of intention, self-regulation and behavioural prepotency was found to differ according to the participants' response to the environmental context. This novel finding was further sustained by results indicating that the 'supported' and 'unsupported' participants did not significantly differ in ways other than their responsiveness to the environmental context. As expected, 'supported' participants demonstrated greater success in translating their intentions into a healthy lifestyle compared with 'unsupported' participants. Although cue saliency was beyond the scope of this study, it is plausible that the most salient cues for 'supported' participants were health-promoting cues whereas 'unsupported' participants may have experienced an attentional bias toward distractions.

Of particular interest is the finding that individual differences in environmental responsiveness affected the factors associated with intention-behaviour consistency. For those supported by environmental cues, only behavioural prepotency predicted future behaviour, above self-regulation and intention. In contrast, both self-regulation and behavioural prepotency were predictive of a healthy lifestyle for 'unsupported' participants. Individuals' responsiveness to cues is shaped by their current needs, learning history and genetics (Hofmann, Friese, \& Wiers, 2008). It may be that past experience alters the cue saliency such that a weak cue, normally insufficient to trigger a behavioural response, may be sufficient to elicit a relapse in an ex-smoker, alcoholic or over-eater due to the strong hedonic response (Schmid, Pratt, \& Howze, 1995). These results support the suggestion that it is an individual's perception of the environment rather than the specifics of the environment that 
determines its influence on behaviour (Kremers, de Bruijn, Visscher, van Mechelen, \& Brug, 2006).

The inclusion of environmental cues within TST is advantageous as the identification and measurement of responses to cues enables a more informed and comprehensive model of health behaviour (Hall \& Fong, 2010; Hofmann, et al., 2008). Further exploration of the association between behavioural prepotency, habits and environmental cues will improve the understanding of behavioural prepotency and the utility of this variable within. In addition, the inclusion of environmental responsiveness indicates the theory's utility for behaviour change (Sallis, 2010), as this variable could identify specific opportunities for interventions. Further, environmental responsiveness confirms how the perceived accessibility of an option is of greater influence to behavioural performance than motivation, self-control or attitudes for 'unsupported' individuals as distractions made the translation of intention into behaviour far more effortful than for 'supported' individuals (Schmid, et al., 1995).

This study examined three objective measures of self-regulation: planning, response inhibition and decision-making. The tasks did not significantly correlate, providing further evidence that self-regulation is multifaceted (Suchy, 2009). 'Unsupported' participants with better planning demonstrated greater consistency in acting upon their intentions to maintain a healthy lifestyle. Literature supports the role of planning in breakfast consumption (Wong \& Mullan, 2009), F\&V consumption (Allan, et al., 2011) and exercise (Sniehotta, Schwarzer, Scholz, \& Schuz, 2005) as good planners appear better able to plan spontaneously and with less effort, supporting their pursuit of goals (Allan, et al., 2011). As expected, 'unsupported' participants with better response inhibition had greater success in translating their intentions into behaviour than those with poorer response inhibition, as they may have been adequately equipped to override prepotent responses and resist temptation in order to successfully maintain a healthy lifestyle (Hall, et al., 2006; Williams \& Thayer, 2009). This is consistent 
with previous research that has found response inhibition is associated with health-risk behaviours including smoking, alcohol consumption and sleep difficulties (Hall, et al., 2006), unintentional eating (Allan, Johnston, \& Campbell, 2010) as well as health-protective behaviours with non-immediate contingencies (Hall \& Fong, 2007).

Whilst health behaviours are reliant on decision-making processes, IGT scores as an executive functioning measure of rational decision-making did not significantly predict variance in lifestyle. This task was designed to assess neurocognitive impairment in decisionmaking and may not have the appropriate sensitivity to detect differences in non-clinical populations. Further, as executive functioning tasks rely upon cognitive processes such as working memory and draw on executive functioning processes other than the one specifically being tested, test results should be interpreted with caution (Miyake et al., 2000). Alternatively, due to the range of behaviours in this study and their repetitive nature, the associated decision-making processes may be more reliant on heuristics rather than conscious deliberation.

In terms of future research, self-regulatory abilities appear responsive to interventions (Baumeister, Gailliot, DeWall, \& Oaten, 2006; Diamond, Barnett, Thomas, \& Munro, 2007), suggesting that intention-behaviour translation for 'unsupported' individuals could be improved. The association between self-regulation and health behaviours appears to be dynamic and bi-directional (Williams \& Thayer, 2009). Regular participation in an exercise program was recently shown to produce significant improvements across a range of regulatory behaviours, including increasing health-promoting behaviours and decreasing health-risk behaviours (Oaten \& Cheng, 2010). These findings are promising for the development of multiple-behaviour change interventions and for improving 'unsupported' individuals' self-regulation. 
TST is an emerging theory, likely to require further refinement as empirical evidence informs the understanding of concepts, their measurement and their interrelationships (Hall \& Fong, 2007). The behavioural prepotency construct has been criticised for not providing insight to the causes of previous actions or opportunities to alter future behaviour (Ajzen, 2002). However, as it is possible to induce and increase behaviour strength through changes to the available cues and through strategies such as implementation intentions, future research could explore if these changes could assist 'supported' individuals to maintain a healthy lifestyle (Brug, de Vet, de Nooijer, \& Verplanken, 2006).

There were methodological limitations to the current study. As with most studies of health behaviour using TST, self-report measures may have affected the accuracy of information obtained due to impression management or distorted recall (Armitage \& Conner, 2001; Hall et al., 2008). However, online and confidential completion of the study may have mitigated the perceived importance of socially desirable responses. This study used a oneweek time frame, meaning the long-term efficacy of these factors could not be determined. However, this timeframe increases the accuracy of self-report measures by reducing recall distortion. Measuring intention and behavioural performance in close proximity is thought to maximise the accuracy of behavioural prediction and allow for the accurate measurement of post-intentional variables within a relatively stable context (Conner \& Godin, 2007; Hall \& Fong, 2007). In addition, it is worth noting that although environmental responsiveness is described as dichotomous by Hall and Fong (2007), creating a dichotomy may influence the power of the power of the results found, and therefore must be interpreted with caution. Finally, the current study examined the performance of a healthy lifestyle by undergraduate students at an Australian university and this may limit the generalisability for the study's findings, as the sample may not be demographically representative of the wider population. Further research with young adults is required to determine if the behavioural patterns of 
undergraduates are representative of general young adult population in order to appropriately inform intervention development.

TST may provide a useful framework for the development of interventions that can influence the behavioural choices made. Interventions to improve the lifestyles of individuals who perceive the environment as supportive should focus on modifying the influence of behavioural prepotency on future behaviour by altering habit strength. If an individual successfully performs a specific health-promoting behaviour, this experience of success through behavioural prepotency creates an impetus to repeat the behaviour. This may in turn lead to positive changes in other health behaviours (de Vries, Kremers, Smeets, \& Reubsaet, 2008). Alternatively, a high degree of motivation is required to overcome behavioural prepotency's powerful influence for health-risk behaviours (Hall \& Fong, 2007). It is arguably unrealistic to expect individuals to make significant behaviour changes in order to maintain a healthy lifestyle if this practice appears to be discouraged by the environment and existing social norms (Schmid, et al., 1995). For example, unhealthy dietary choices are widely promoted and accessible, with the marketing of these options optimising their desirability and prominence (Hall \& Fong, 2007). Restructuring the environment in order to increase the accessibility and convenience of health-promoting choices and simultaneously decreasing tempting alternatives may improve the translation of intentions into behaviour. Additionally, this would decrease the demands on individuals' self-regulatory capacity, as there would be less need to inhibit responses to distractions (Hall, et al., 2006).

The current study identifies environmental responsiveness as a possible important determinant to close the intention-behaviour gap for maintaining a healthy lifestyle. Individuals who perceive their environment as supportive act on their intentions with greater ease than those unsupported by their environment. Improving the behavioural strength of health-promoting behaviours will arguably further improve the translation of intention into 
behaviour for 'supported' individuals. While this will also improve the intention-behaviour consistency for 'unsupported' individuals, further measures such as reducing environmental distractions, improving the accessibility of health-promoting choices and increasing their self-regulatory abilities are required for these individuals. Intervening before their lifestyles become stable and more resistant to change will optimise the opportunities for success and arguably lead to a better quality of life and reduction in risk for chronic illness. 


\section{References}

Abraham, C., Sheeran, P., Norman, P., Conner, M., De Vries, N., \& Otten, W. (1999). When good intentions are not enough: Modeling postdecisional cognitive correlates of condom use. Journal of Applied Social Psychology, 29(12), 2591-2612.

ABS. (2006). Tobacco smoking in Australia: A snapshot, 2004-2005. Canberra.

Adams, T. B., \& Colner, W. (2008). The association of multiple risk factors with fruit and vegetable intake among a nationwide sample of college students. Journal of American College Health, 56(4), 455-461.

AIFW. (2011). Young Australians: Their health and wellbeing 2011 (No. Cat no. PHE 140). Canberra: Australian Institute of Health and Welfare.

Ajzen, I. (1991). The theory of planned behavior. Organizational Behavior and Human Decision Processes, 50, 179-211.

Ajzen, I. (2002). Residual effects of past on later behavior: Habituation and reasoned action perspectives. Personality and Social Psychology Review, 6(2), 107-122.

Allan, J. L., Johnston, M., \& Campbell, N. (2010). Unintentional eating. What determines goal-incongruent chocolate consumption? Appetite, 54, 422-425.

Allan, J. L., Johnston, M., \& Campbell, N. (2011). Missed by an inch or a mile? Predicting the size of intention-behaviour gap from measures of executive control. Psychology \& Health, 26(6), 635-650.

Allom, V., \& Mullan, B. (2011). Self-regulation versus habit: The influence of self-schema on fruit and vegetable consumption. Psychology \& Health.

Armitage, C., \& Conner, M. (2001). Efficacy of the theory of planned behaviour: A metaanalytic review. British Journal of Social Psycholgy, 40, 471-499. 
Baumeister, R. F., Gailliot, M. T., DeWall, C. N., \& Oaten, M. (2006). Self-regulation and personality: How interventions increase regulatory success, and how depletion moderates the effects of traits on behavior. Journal of Personality, 74, 1773-1800.

Baumeister, R. F., Schmeichel, B. J., \& Vohs, K. D. (2007). Self-regulation and the executive function: The self as controlling agent. In A. W. Kruglanski \& E. T. Higgins (Eds.), Social psychology: Handbook of basic principles (2nd ed.).

Belloc, N. B., \& Breslow, L. (1972). Relationship of physical health status and health practices. Preventive Medicine, 1, 409-421.

Berkman, L. F., \& Syme, S. L. (1979). Social networks, host resistance, and mortality: A nine-year follow-up study of Alameda County residents. American Journal of Epidemiology, 109(2), 186-204.

Brock, B. M., Haefner, D. P., \& Noble, D. S. (1988). Alameda County redux: Replication in Michigan. Preventive Medicine, 17, 483-495.

Brug, J., de Vet, E., de Nooijer, J., \& Verplanken, B. (2006). Predicting fruit consumption: Cognitions, intention and habit. Journal of Nutrition Education and Behavior, 38, 73 81.

Collins, A., \& Mullan, B. (2011). An extention of the theory of planned behavior to predict immediate hedonic behaviors and distal benefit behaviors. Food Quality and Preference.

Conner, M., \& Godin, G. (2007). Temporal stability of behavioural intention as a moderator of intention-health behaviour relationships. Psychology \& Health, 22(8), 875-897.

de Vries, H., Kremers, S., Smeets, T., \& Reubsaet, A. (2008). Clustering of diet, physical activity and a general willingness to change. Psychology \& Health, 23(3), 265-278.

Diamond, A., Barnett, W. S., Thomas, J., \& Munro, S. (2007). Preschool program improves cognitive control. Science, 318, 17151-17156. 
Dodd, L. J., Al-Nakeeb, Y., Nevill, A., \& Forshaw, M. J. (2010). Lifestyle risk factors of students: A cluster analytical approach. Preventive Medicine, 51, 73-77.

Hall, P. A., Elias, L. J., \& Crossley, M. (2006). Neurocognitive influences on health behavior in a community sample. Health Psychology, 25(6), 778-782.

Hall, P. A., Elias, L. J., Fong, G. T., Harrison, A. H., Bowrowsky, R., \& Sarty, G. E. (2008). A social neuroscience perspective on physical activity. Journal of Sport \& Exercise Psychology, 30, 432-449.

Hall, P. A., \& Fong, G. T. (2007). Temporal self-regulation theory: A model for individual health behavior. Health Psychology Review, 1(1), 6-52.

Hall, P. A., \& Fong, G. T. (2010). Temporal self-regulation theory: Looking forward. Health Psychology Review, 4(2), 83-92.

Hofmann, W., Friese, M., \& Wiers, R. W. (2008). Impulsive versus reflective influences on health behavior: A theoretical framework and empirical review. Health Psychology Review, 2(2), 111-137.

Kaplan, G. A., Baltrus, P. T., \& Raghunathan, T. E. (2007). The shape of health to come: Prospective study of the determinants of 30-year health trajectories in the Alameda County study. International Journal of Epidemiology, 36, 542-548.

Keller, S., Maddock, J. E., Hannover, W., Thyrian, J. R., \& Basler, H. (2008). Multiple health risk behaviors in German first year university students. Preventive Medicine, 46, 189195.

Kor, K., \& Mullan, B. (2011). Sleep hygiene behaviours: An application of the theory of planned behaviour and the investigation of perceived autonomy support, past behaviour and response inhibition. Psychology \& Health, iFirst, 1-17. 
Kremers, S., de Bruijn, G., Visscher, T., van Mechelen, W., \& Brug, J. (2006).

Environmental influences on energy balance-related behaviors: A dual-process view. International Journal of Behavioral Nutrition and Physical Activity, 3(9).

Kwan, M. Y. W., Bray, S. R., \& Ginis, K. A. M. (2009). Predicting physical activity of firstyear university students: An application of the theory of planned behavior. Journal of American College Health, 58(1), 45-55.

Lippke, S., \& Ziegelmann, J. P. (2008). Health behavior and health behavior change- theories and evidence. Applied Psychology, 57(4), 541-543.

Michie, S., Rothman, A. J., \& Sheeran, P. (2007). Current issues and new direction in Psychology and Health: Advancing the science of behavior change. Psychology \& Health, 22(3), 249-253.

Milligan, R. A. K., Burke, V., Beilin, L. J., Richards, J., Dunbar, D., Spencer, M., et al. (1998). Health-related behaviours and psycho-social characteristics of 18 year-old Australians. Social Science and Medicine, 45(10), 1549-1562.

Miyake, A., Friedman, N. P., Emerson, M. J., Witzki, A. H., Howerter, A., \& Wager, T. D. (2000). The unity and diversity of executive functions and their contributions to complex "frontal lobe" tasks: A latent variable analysis. Cognitive Psychology, 41(1), 49-100.

Mullan, B., Wong, C., Allom, V., \& Pack, S. L. (2011). The role of executive function in bridging the intention-behaviour gap for binge-drinking in university students. Addictive Behaviors.

Norman, P., \& Conner, M. (2006). The theory of planned behaviour and binge drinking: Assessing the moderating role of past behaviour within the theory of planned behaviour. British Journal of Health Psychology, 11, 55-70. 
Oaten, M., \& Cheng, K. (2010). Longitudinal gains in self-regulation from regular physical exercise. British Journal of Health Psychology, 11, 717-733.

Ouellette, J. A., \& Wood, W. (1998). Habit and intention in everyday life: The multiple processes by which past behavior predicts future behavior. Psychological Bulletin, 124(1), 54-74.

Pearson, N., Atkin, A. J., Biddle, S. J. H., Gorely, T., \& Edwardson, C. (2009). Patterns of adolescent physical activity and dietary behaviours. International Journal of Behavioral Nutrition and Physical Activity, 6.

Quintiliani, L., Allen, J., Marino, M., Kelly-Weeder, S., \& Li, Y. (2010). Multiple health behavior clusters among female college students. Patient Education and Counseling, 79, 134-137.

Sallis, J. F. (2010). Temporal self-regulation theory: A step forward in the evolution of health behaviour models. Health Psychology Review, 4(2), 75-78.

Schmid, T. L., Pratt, M., \& Howze, E. (1995). Policy as intervention: Environmental and policy approaches to the prevention of cardiovascular disease. American Journal of Public Health, 85(9), 1207-1211.

Schweiger, A., Abramovitch, A., Doniger, G. M., \& Simon, E. S. (2007). A clinical construct validity study of a novel computerised battery for the diagnosis of ADHD in young adults. Journal of Clinical and Experimental Neuropsychology, 29(1), 100-111.

Shallice, T. (1982). Specific Impairments of Planning. Philosophical Transactions of the Royal Society of London. B, Biological Sciences, 298(1089), 199-209.

Sheeran, P., \& Abraham, C. (2003). Mediator of moderators: Temporal stability of intention and the intention-behavior relation. Personality and Social Psychology Bulletin, 29(2), 205-215. 
Sniehotta, F. F., Schwarzer, R., Scholz, U., \& Schuz, B. (2005). Action planning and coping planning for long-term lifestyle change: Theory and assessment. European Journal of Social Psychology, 35, 565-576.

Suchy, Y. (2009). Executive functioning: Overview, assessment and research issues for nonneuropsychologists. Annals of Behavioural Medicine, 37, 106-116.

Sutton, S. (1998). Predicting and Explaining Intentions and Behavior: How Well Are We Doing? Journal of Applied Social Psychology, 28(15), 1317-1338.

Todd, J., Brogan, A., Mullan, B., \& Carroll, L. (under review). The internal and environmental cues to action scale (CAS): Towards a better understanding of behavioural prepotency.

Todd, J., \& Mullan, B. (2011). Using the theory of planned behaviour and prototype willingness model to target binge drinking in female undergraduate university students. Addictive Behaviors, 36, 980-986.

Webb, T. L., \& Sheeran, P. (2006). Does changing behavioural intentions engender behavior change? A meta-analysis of the experimental evidence Psychological Bulletin, 132(2), 249-268.

WHO. (2003). Diet, nutrition and the prevention of chronic diseases. Geneva.

WHO. (2011). Global status report on noncommunicable diseases 2010. Geneva: World Health Organisation.

Williams, P. G., \& Thayer, J. F. (2009). Executive functioning and health: Introduction to the special series. Annals of Behavioural Medicine, 37(2), 101-105.

Wong, C., \& Mullan, B. (2009). Predicting breakfast consumption: An application of the theory of planned behaviour and the investigation of past behaviour and executive function. British Journal of Health Psychology, 14, 489-504. 


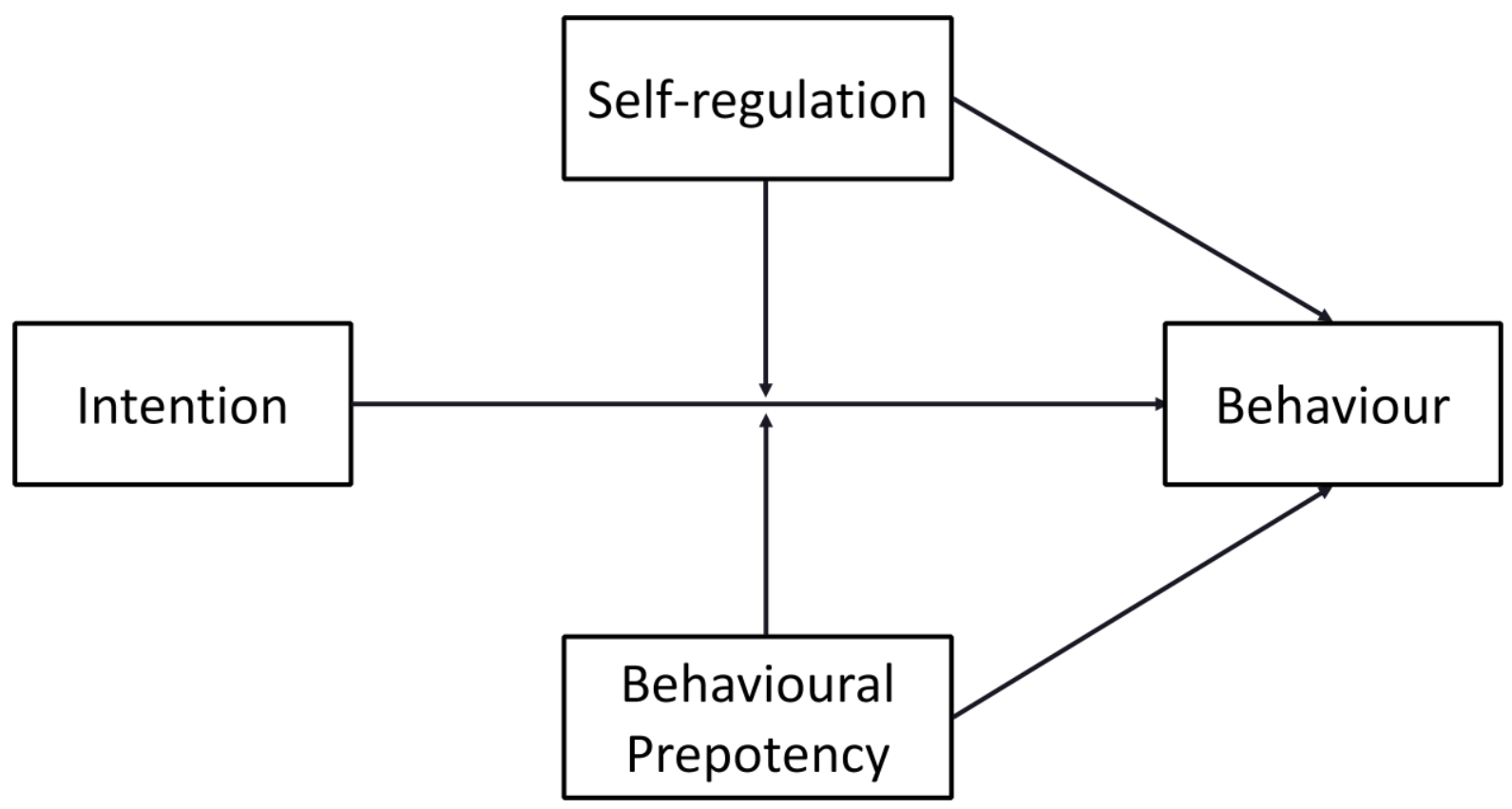

Figure 1. Schematic representation of the Temporal Self-Regulation Theory(Hall \& Fong, 2007)(Hall \& Fong, 2007)(Hall \& Fong, 2007)(Hall \& Fong, 2007)(Hall \& Fong, 2007)(Hall \& Fong, 2007)(Hall \& Fong, 2007)(Hall \& Fong, 2007) 


\section{Table 1}

Behaviour measures and guidelines

\section{Behaviour}

Physical activity

Fruit and vegetable

consumption

Breakfast consumption

Sleep

Alcohol consumption

$$
\text { injury }
$$

Smoking
- Less than two standard drinks on any day.

- No more than four standard drinks on a single occasion

\section{Australian National Guidelines}

Thirty minutes of moderate intensity activity on most, if not

all days

Two pieces of fruit and five serves of vegetables

Daily consumption of breakfast

Eight hours on average per night

For the reduction of lifetime risk and risk of alcohol-related

Avoidance of smoking 
Table 2

Pearson's product correlations of individual health behaviours

\begin{tabular}{|c|c|c|c|c|c|c|}
\hline & PA & $\mathrm{F} \& \mathrm{~V}$ & Breakfast & Sleep & Alcohol & Smoking \\
\hline$\overline{\mathrm{PA}}$ & - & $.227 * *$ & $.278 * *$ & .066 & .107 & .100 \\
\hline $\mathrm{F} \& \mathrm{~V}$ & & - & $.281 * *$ & -.069 & -.004 & .022 \\
\hline Breakfast & & & - & .043 & .136 & .111 \\
\hline Sleep & & & & - & .030 & .024 \\
\hline Alcohol & & & & & - & $.623 * *$ \\
\hline Smoking & & & & & & - \\
\hline
\end{tabular}

Note $. \mathrm{PA}=$ physical activity, $\mathrm{F} \& \mathrm{~V}=$ fruit and vegetable consumption. ${ }^{* *}$ denotes statistical significance at the 0.01 level (2-tailed). 
Table 3

Pearson product correlations, means and standard deviations of predictors of healthy lifestyle behaviour in unsupportive and supportive environmental contexts

\begin{tabular}{lrrrrrrr}
\hline & Intention & BP & GNG & IGT Behaviour & M & SD \\
\hline Intention & - & $.534^{* *}$ & -.184 & .007 & $.355^{* *}$ & 3.47 & 0.92 \\
BP & $.317^{* *}$ & - & -.138 & -.092 & $.583^{* *}$ & 3.62 & 1.23 \\
GNG & .073 & .021 & - & .056 & .184 & 12.44 & 0.06 \\
IGT & -.078 & .030 & -.015 & - & .038 & 1769.44 & 698.21 \\
Behaviour & $.323^{* *}$ & $.712^{* *}$ & .103 & .085 & - & 3.38 & 1.30 \\
M & 3.71 & 3.60 & 12.40 & 1702.66 & 3.46 & - & \\
SD & 0.84 & 1.48 & 0.15 & 640.71 & 1.51 & & - \\
\hline
\end{tabular}

Note. Correlations, means, and standard deviations above the diagonal are for unsupportive environments; correlations, means, and standard deviations below the diagonal are for supportive environments.

$\mathrm{BP}=$ behavioural preopotency. Self regulation measures are GNG (Go NoGo performance index) and IGT (Iowa gambling task final amount). ** denotes statistical significance at the 0.01 level (2-tailed) 


\section{Table 4}

Hierarchical regression analysis: intention, environmental responsiveness, self-regulation and behavioural prepotency as predictors of HLS

\begin{tabular}{|c|c|c|c|c|c|c|}
\hline & & B & $t$ & $R^{2}$ & $\Delta R^{2}$ & $p$ \\
\hline \multirow[t]{2}{*}{1} & INT & .351 & 4.420 & & & $.000 * *$ \\
\hline & & & & .123 & & $.000 * *$ \\
\hline \multirow[t]{3}{*}{2} & INT & .317 & 3.931 & & & $.000 * *$ \\
\hline & ET & .159 & 1.972 & & & .051 \\
\hline & & & & .147 & .024 & .051 \\
\hline \multirow[t]{6}{*}{3} & INT & .324 & 3.969 & & & $.000 * *$ \\
\hline & ET & .167 & 2.066 & & & $.041 *$ \\
\hline & IGT & .078 & .981 & & & .328 \\
\hline & TOL & .047 & .581 & & & .562 \\
\hline & GNG & .091 & 1.158 & & & .249 \\
\hline & & & & .166 & .019 & .249 \\
\hline \multirow[t]{7}{*}{4} & INT & .069 & .973 & & & .332 \\
\hline & ET & .140 & 2.186 & & & $.031 *$ \\
\hline & IGT & .065 & 1.027 & & & .306 \\
\hline & TOL & .055 & .853 & & & .395 \\
\hline & GNG & .107 & 1.701 & & & .091 \\
\hline & BP & .618 & 8.946 & & & $.000 * *$ \\
\hline & & & & .478 & .312 & $.000 * *$ \\
\hline
\end{tabular}

Note. Dependent variable: HLS. INT=intention, ET=environmental responsiveness score, $\mathrm{IGT}=$ decision-making measure, $\mathrm{TOL}=$ planning measure, $\mathrm{GNG}=$ response inhibition measure, $\mathrm{BP}=$ behavioural prepotency, HLS=Healthy Lifestyle Score. ** denotes statistical significance at the .01 level. * denotes statistical significance at the .05 level. 


\section{Table 5}

Final block of the hierarchical regression analysis: TST variables as predictors of HLS within 'supported' participants.

\begin{tabular}{|c|c|c|c|c|c|c|}
\hline Block & & $B$ & $t$ & $R^{2}$ & $\Delta R^{2}$ & $p$ \\
\hline \multirow[t]{4}{*}{3} & INT & .092 & 1.184 & & & .239 \\
\hline & TOL & -.032 & -.434 & & & .665 \\
\hline & BP & .677 & 8.676 & & & $.000 * *$ \\
\hline & & & & .518 & .398 & $.000 * *$ \\
\hline \multirow[t]{4}{*}{3} & INT & .124 & 1.564 & & & .121 \\
\hline & GNG & .080 & 1.078 & & & .284 \\
\hline & BP & .653 & 8.260 & & & $.000 * *$ \\
\hline & & & & .508 & .373 & $.000 * *$ \\
\hline \multirow[t]{4}{*}{3} & INT & .114 & 1.492 & & & .139 \\
\hline & IGT & .074 & 1.013 & & & .314 \\
\hline & BP & 677 & 8.852 & & & $.000 * *$ \\
\hline & & & & .528 & .411 & $.000 * *$ \\
\hline
\end{tabular}

Note. Dependent variable: HLS. INT=intention, TOL= planning measure, GNG=response inhibition measure, IGT=decision making measure, $\mathrm{BP}=$ behavioural prepotency, HLS= Healthy Lifestyle Score. ** denotes statistical significance at the .001 level. 


\section{Table 6}

Final block of the hierarchical regression analysis: TST variables as predictors of HLS within 'unsupported' participants.

\begin{tabular}{|c|c|c|c|c|c|c|}
\hline Block & Variable & $\beta$ & $t$ & $R^{2}$ & $\Delta R^{2}$ & $p$ \\
\hline \multirow[t]{4}{*}{3} & INT & .101 & .786 & & & .435 \\
\hline & TOL & .262 & 2.416 & & & $.019 *$ \\
\hline & $\mathrm{BP}$ & .542 & 4.255 & & & $.000 * *$ \\
\hline & & & & .410 & .210 & $.000 * *$ \\
\hline \multirow[t]{4}{*}{3} & INT & .090 & .683 & & & .498 \\
\hline & GNG & .277 & 2.443 & & & $.018 *$ \\
\hline & $\mathrm{BP}$ & .554 & 4.262 & & & $.000 * *$ \\
\hline & & & & .392 & .225 & $.000 * *$ \\
\hline \multirow[t]{4}{*}{3} & INT & .059 & .424 & & & .673 \\
\hline & IGT & .088 & .753 & & & .455 \\
\hline & BP & .542 & 3.896 & & & $.000 * *$ \\
\hline & & & & .290 & .203 & $.000 * *$ \\
\hline
\end{tabular}

Note. Dependent variable: HLS. INT=intention, TOL= planning measure, GNG=response inhibition measure, IGT=decision making measure, $\mathrm{BP}=$ behavioural prepotency, HLS= Healthy Lifestyle Score.

** denotes statistical significance at the .001 level, * denotes statistical significance at the .05 level. 